\title{
Aberrant DNA methylation of the PDGF gene in homocysteine-mediated VSMC proliferation and its underlying mechanism
}

\author{
XUE-BO HAN $^{1 *}$, HUI-PING ZHANG ${ }^{2 *}$, CHENG-JIAN CAO ${ }^{1}$, YAN-HUA WANG $^{1}$, JUE TIAN $^{3,4}$, \\ XIAO-LING YANG ${ }^{3,4}$, AN-NING YANG $^{3}$, JIE WANG ${ }^{5}$, YI-DENG JIANG ${ }^{3}$ and HUA XU ${ }^{3,4}$ \\ ${ }^{1}$ Department of Laboratory Medicine, Ningxia Medical University, ${ }^{2}$ Department of Prenatal Diagnosis Center, \\ General Hospital of Ningxia Medical University, ${ }^{3}$ Department of Pathophysiology, Basic Medical School, \\ Ningxia Medical University, ${ }^{4}$ Key Laboratory of Basic Research in Cardio-Cerebral Vascular Diseases, \\ ${ }^{5}$ Center of Scientific Technology, Ningxia Medical University, Yinchuan, \\ Ningxia Hui Autonomous Region 750004, P.R. China
}

Received August 7, 2013; Accepted April 8, 2014

DOI: $10.3892 / \mathrm{mmr} .2014 .2249$

\begin{abstract}
It is well established that homocysteine (Hcy) is an independent risk factor for atherosclerosis (AS), which is characterized by vascular smooth muscle cell (VSMC) proliferation. However, the molecular mechanism underlying AS in VSMCs is yet to be elucidated. The aim of this study was to investigate the potential involvement of aberrant DNA methylation of the platelet-derived growth factor (PDGF) gene in Hcy-mediated VSMC proliferation and its underlying mechanism. Cultured human VSMCs were treated with varying concentrations of Hcy. VSMC proliferation, PDGF mRNA and protein expression and PDGF promoter demethylation showed a dose-dependent increase with Hcy concentration, suggesting an association among them. Cell cycle analysis revealed a decreased proportion of VSMCs in $\mathrm{G}_{0} / \mathrm{G}_{1}$ and an increased proportion in $\mathrm{S}$ phase, indicating that VSMC proliferation was increased under Hcy treatment. Furthermore, S-adenosylhomocysteine (SAH) levels were observed to increase and those of $\mathrm{S}$-adenosylmethionine (SAM) were observed to decrease. The consequent decrease in the ratio of SAM/SAH may partially explain the hypomethylation of PDGF with Hcy treatment. Folate treatment
\end{abstract}

Correspondence to: Dr Yi-Deng Jiang, Department of Pathophysiology, Basic Medical School, Ningxia Medical University, 1160 Shengli Street, Yinchuan, Ningxia Hui Autonomous Region 750004, P.R. China

E-mail: xuecca@163.com

*Contributed equally

Key words: homocysteine, vascular smooth muscle cell proliferation, platelet-derived growth factor, methylation, methionine cycle, atherosclerosis exhibited an antagonistic effect against Hcy-induced VSMC proliferation, aberrant PDGF methylation and PDGF expression. These data suggest that Hcy may stimulate VSMC proliferation through the PDGF signaling pathway by affecting the epigenetic regulation of PDGF through the demethylation of its promoter region. These findings may provide novel insight into the molecular association between aberrant PDGF gene demethylation and the proliferation of VSMCs in Hcy-associated AS.

\section{Introduction}

Vascular smooth muscle cells (VSMCs) are one of the major types of cell in the blood vessel, and their proliferation is associated with cardiovascular diseases and atherosclerosis (AS) (1). Numerous clinical and experimental studies have investigated the pathogenic mechanism underlying VSMC proliferation (2-5) in order to identify potential therapeutic targets for VSMC proliferation in cardiovascular diseases. However, at present, such targets are yet to be elucidated.

Evidence has revealed a marked stimulatory effect of homocysteine (Hcy) on VSMC proliferation (6). Hcy has been reported to be an independent risk factor for AS (7), and mild to moderate increases in Hcy serum levels, which may be due to nutritional or genetic factors, are frequently observed in patients with AS; therefore, the stimulatory effect of Hcy on VSMC proliferation may be highly involved in AS pathogenesis. The identification of the molecular mechanisms underlying Hcy-induced VSMC proliferation may be particularly beneficial.

Hcy is a non-protein, sulfur-containing amino acid, which is formed exclusively upon demethylation of S-adenosylmethionine (SAM), the active form of methionine. SAM serves as the primary methyl donor for $>100$ transmethylation reactions, including DNA methylation modification (8). Following the transfer of its methyl group, SAM is converted to $\mathrm{S}$-adenosylhomocysteine (SAH), which is a potent inhibitor of SAM-dependent methyltransferases (9). SAH then under- 
goes hydrolysis to form Hcy. In the methionine cycle, Hcy is remethylated to generate methionine and further activated to produce SAM. Hcy may also undergo hydrolysis. Increased Hcy levels may interfere with the methionine cycle and, consequently, DNA methylation modification.

Modification of DNA methylation is the primary mechanism of epigenetic gene regulation (10). Generally, hypermethylation inhibits, while hypomethylation promotes, gene expression (11). The involvement of aberrant DNA methylation has been confirmed in the pathogenesis of various diseases, including cancer and AS (12). Our previous study showed that Hcy-induced aberrant DNA methylation patterns in AS affect numerous genes, including peroxisome proliferator-activated receptor $\alpha$ and apolipoprotein E (13). However, the methylation pattern of the platelet-derived growth factor (PDGF) gene, which is a potent mitogen for VSMC proliferation, is yet to be elucidated in Hcy-treated VSMCs.

PDGF has significant roles in developmental and physiological processes, and has also been implicated in various proliferative disorders. PDGF has been shown to be a potent stimulator of VSMC growth (2). Furthermore, PDGF has been identified as an important mediator of VSMC proliferation, with potential mechanisms including activation of the Ras pathway, phosphoinositol 3'-kinase, promotion of VSMC extracellular $\mathrm{Ca}^{2+}$ influx and release of intracellular $\mathrm{Ca}^{2+}$ (14-17). The impact of Hcy on the epigenetic regulation of PDGF, which may influence PDGF expression and be involved in VSMC proliferation, may provide novel insight into the pathogenesis of AS.

In the present study, Hcy-induced VSMC proliferation, PDGF expression and the methylation pattern of the PDGF gene were investigated. Furthermore, their causative correlation and the potential mechanism were also investigated, in order to identify a useful target for the prevention and treatment of AS induced by Hcy.

\section{Materials and methods}

Cell culture. The study was approved by the ethics committee of Ningxia Medical University (Yinchuan, China). Primary cultured VSMCs were obtained from human umbilical vein media (Department of Obstetrics, General Hospital of Ningxia Medical University, Yinchuan, China). Cells were cultured in Dulbecco's Modified Eagle's medium-Ham's F12 media (Gibco-BRL, Gaithersburg, MD, USA) supplemented with $20 \%$ fetal calf serum (FCS; Gibco-BRL), $100 \mathrm{U} / \mathrm{ml}$ penicillin and streptomycin (Sigma-Aldrich Trading Co., Ltd, Shanghai, China) at $37^{\circ} \mathrm{C}$ in an incubator with $5 \% \mathrm{CO}_{2}$. The purity of the primary VSMC culture was confirmed by the characteristic 'hill-and-valley' growth pattern and immunocytochemistry of $\alpha$-actin. VSMCs were used at between three and five passages for the experiment. Cells were seeded onto six-well plates and grown to $80 \%$ confluence. Cells were then serum-deprived for $24 \mathrm{~h}$ to reach synchrony, followed by the addition of $5 \%$ FCS for a further $24 \mathrm{~h}$ and the administration of Hcy (Sigma-Aldrich Trading Co., Ltd). Hcy was administered at the following concentrations: 0 (control), 50, 100, 200 and $500 \mu \mathrm{M}$ and $500 \mu \mathrm{M}$ plus folate (Sigma-Aldrich Trading Co., Ltd), respectively. Hcy was replenished every $8 \mathrm{~h}$ in compensation for its short half-life (total three times).
Cell viability assay. To assess cell proliferation, VSMCs were seeded in 96 -well plates at $10^{3}-10^{4}$ cells in $100 \mu \mathrm{l} /$ well. The aforementioned concentrations of Hcy were then added and the cells were incubated for $72 \mathrm{~h}$. Hcy was replenished every $8 \mathrm{~h}$. A total of $20 \mu \mathrm{l} \mathrm{MTT}(5 \mathrm{mg} / \mathrm{ml}$; Sigma-Aldrich Trading Co., Ltd) was added to each well and incubated at $37^{\circ} \mathrm{C}$ for $4 \mathrm{~h}$. The supernatant was removed using a pipette, and $150 \mu \mathrm{l}$ dimethyl sulfoxide was added to each well. After $10 \mathrm{~min}$ of incubation at room temperature, plates were read on a micro-enzyme-linked immunosorbent assay reader (Bio-Tek Instruments, Inc., Winooski, VT, USA) at $490 \mathrm{~nm}$. Values were normalized using the control value.

Flow cytometric analysis. Following treatment with various concentrations of Hcy, cells were analyzed using a FACStar ${ }^{\mathrm{TM}}$ Plus flow cytometer (Becton, Dickinson and Company, Franklin Lakes, NJ, USA). In brief, cells were washed once with phosphate-buffered saline (PBS; $0.01 \mathrm{~mol} / \mathrm{l}, \mathrm{pH}$ 7.2) and fixed at $-10^{\circ} \mathrm{C}$ for $5 \mathrm{~min}$ in paraformaldehyde-lysine-periodate fixation solution. Cells were then treated with $0.1 \%$ Triton X-100 and $0.5 \%$ RNase A, followed by the addition of propidium iodide. All samples were passed through 70-mm mesh prior to flow cytometric analysis; 30,000 nuclei were examined in each analysis. The percentage of cells was estimated using the Cell FIT analysis version 2.0 software (Becton, Dickinson and Company). Three separate experiments were performed with three different populations of cells.

Quantitative polymerase chain reaction ( $q P C R$ ). Total RNA was isolated using $\mathrm{TRIzol}^{\circledR}$ reagent (Invitrogen Life Technologies, Grand Island, NY, USA) and reverse transcribed using the RevertAid First Strand cDNA Synthesis kit (MBI Fermentas, Vilnius, Lithuania). GAPDH was used as an endogenous control, and the primer sequences were as follows: PDGF, 5'-TCTGCTGCTACCTGCGTCTGG-3' (forward) and 5'-CACTGCACGTTGCGGTTGTT-3' (reverse) and GAPDH, 5'-AGAAGGCTGGGGCTCATTTG-3' (forward) and 5'-AGGGGCCATCCACAGTCTTC-3' (reverse). qPCR analysis was performed using an FTC-3000 Real-Time PCR detection system (Funglyn Biotech Corp. Ltd., Toronto, ON, Canada). The RNA levels of each gene were calculated using the cycle threshold $(\mathrm{Ct})$ value of the sample relative to that of GAPDH using the following formula: $\mathrm{Ct}=\mathrm{Ct}_{(\mathrm{GAPDH})}-\mathrm{Ct}_{\text {(sample) }}$. Final results, expressed as n-fold differences in target gene expression relative to the calibrator, termed $\mathrm{N}_{\text {target }}$, were calculated using the following formula: $\mathrm{N}_{\text {target }}=2^{\mathrm{Ct} \text { (sample)-Ct(calibrator) }}$, where $\mathrm{Ct}$ values of the calibrator and sample were determined by subtracting the $\mathrm{Ct}$ value of the target gene from the $\mathrm{Ct}$ value.

Western blot analysis. Proteins were extracted from the cells using cell lysis buffer and separated using $12 \%$ SDS-PAGE. The proteins and the prestained marker (MBI Fermentas, Amherst, NY, USA) were then transferred onto a polyvinylidene fluoride Immobilon ${ }^{\circledR}$-P Transfer Membrane (Millipore, Billerica, MA, USA) with a pore size of $0.45 \mathrm{~mm}$ using a Trans-Blot ${ }^{\circledR}$ Semi-Dry Transfer Cell model 755 (Bio-Rad, Hercules, CA, USA) for $90 \mathrm{~min}$. The membrane was incubated at room temperature in PBS-Tween 20 (PBS-T) buffer containing $5 \%$ non-fat milk for $4 \mathrm{~h}$. Membranes were then cut as required 
and placed in a hybridization bag with $1 \mathrm{ml}$ anti- $\beta$-actin or -PDGF primary antibodies (Sigma-Aldrich, St. Louis, MO, USA) and incubated at $4^{\circ} \mathrm{C}$ overnight. The membranes were subsequently washed three times using PBS-T buffer and the secondary antibody was added for $2 \mathrm{~h}$ at room temperature. The membranes were washed a further three times with Tris buffered saline with Tween 20 and incubated with horseradish peroxidase substrate (BeyoECL Plus A/B; Beyotime Institute of Biotechnology, Shanghai, China) for $1 \mathrm{~min}$. Blots were developed using X-film (Kodak, Tokyo, Japan).

Detection of PDGF gene methylation using nested methylation-specific (nMS)-PCR. Genomic DNA was isolated from VSMCs using the Wizard ${ }^{\circledR}$ Genomic DNA purification kit (Promega Corp., Madison, WI, USA) and nMS-PCR was performed for the detection of PDGF gene methylation. Following standard sodium bisulfite DNA modification using the EZ DNA Methylation-Gold ${ }^{\mathrm{TM}}$ kit (Zymo Research, Irvine, CA, USA), two-step PCR amplifications were performed. nMS-PCR initially uses an outer primer pair that does not contain any $\mathrm{CpG}$ sites, followed by second step PCR using conventional PCR primers. $\mathrm{CpG}$ islands were identified in the PDGF promoter region using a $\mathrm{CpG}$ Island Search engine (http://www.uscnorris.com/cpgislands2/cpg.aspx; http://www. cbs.dtu.dk/services/promoter/). The primers used in the nMS-PCR assay were as follows: PDGF-outer primer, 5'-TTTTTTT GTTTTGAAATTTTGGTTAA-3' (forward) and 5'-CAAAATCCCAACAAAAAAAATCTCC-3' (reverse); PDGF-methylated primer, 5'-TTTGGAAATTAATGATAA GTTAGGC-3' (forward) and 5'-AAGCATCATAAA AAACAAACGCATC-3' (reverse); PDGF-unmethylated primer, 5'-TTGGAAATTAATGATAAGTTAGGTGA-3' (forward) and 5'-AAACATCATAAAAAACAAACA CATCA-3' (reverse). To reduce mispriming and to increase efficiency, touchdown PCR was used for the amplification. The PCR products were separated using electrophoresis on a $2 \%$ agarose gel containing ethidium bromide. DNA bands were visualized using ultraviolet light and methylation was calculated using the following formula: Methylation $\%=$ methylation/(methylation + unmethylation) x 100 .

SAM and SAH concentrations examined by high-performance liquid chromatography (HPLC). The concentrations of SAM and SAH were determined using HPLC. Cells were centrifuged, washed twice with cold PBS and maintained on ice. The cell pellets were subsequently homogenized in four volumes of $0.4 \mu \mathrm{M} \mathrm{HClO}_{4}$. A $200 \mu \mathrm{l}$ aliquot of the acid extract was loaded into a C18 column (Shimadzu, Kyoto, Japan), run by a Hitachi L2000 HPLC system (Hitachi, Tokyo, Japan). The absorption of the eluted compounds was monitored at an excitation wavelength of $254 \mathrm{~nm}$. Elution of SAM and SAH was achieved at a flow rate of $1.0 \mathrm{ml} / \mathrm{min}$ using mobile-phase ammonium formate solution. Chromatograms were recorded using a D-2000 Elite integrator. SAM and SAH standards were used to identify the elution peaks, and the SAM and SAH tissue values were calculated using the standard curve.

Endogenous C-5 DNA methyltransferase (C-5 MT-ase) activity assay. A modification of the assay developed by Hattori et al (18) was used to determine the activities of DNA methyltransferases. VSMCs $\left(1 \times 10^{6}\right)$ were homogenized using a glass pestle containing $500 \mu \mathrm{l}$ lysis buffer $(100 \mathrm{mmol} / \mathrm{l} \mathrm{NaCl}$, $10 \mathrm{mmol} / \mathrm{l}$ Tris-HCl, pH 8.0, $25 \mathrm{mmol} / \mathrm{l}$ EDTA, 0.5\% SDS and proteinase $\mathrm{K} 0.2 \mathrm{~g} / \mathrm{l})$. The suspension was freeze-thaw cycled between -70 and $37^{\circ} \mathrm{C}$ three times. VSMC protein exacts were stored at $-70^{\circ} \mathrm{C}$ prior to analysis. The reaction contained cell homogenates (5 $\mu \mathrm{g}$ protein), $0.25 \mu \mathrm{g}$ poly(deoxyinosinic-deoxycytidylic) acid and $11.1 \times 10^{10} \% \mathrm{~Bq}$ [methyl- $\left.{ }^{3} \mathrm{H}\right] \mathrm{SAM}$ in a total volume of $20 \mu \mathrm{l}$, and was incubated at $37^{\circ} \mathrm{C}$ for $2 \mathrm{~h}$. RNA was removed by adding $20 \mu \mathrm{l}$ RNase A ( $2 \mathrm{~g} / \mathrm{l})$ and incubating at room temperature for $5 \mathrm{~min}$. DNA was purified using the E.Z.N.A ${ }^{\circledR}$ Cycle-Pure kit (Omega Bio-Tek, Inc., Norcross, GA, USA), and the purified genomic DNA was spotted onto a Whatman $\mathrm{GF} / \mathrm{C}$ filter disc and dried at $80^{\circ} \mathrm{C}$ for $5 \mathrm{~min}$, prior to counting in a Packard 1600 TR Liquid Scintillation Counter (Packard Instrument Co., Meriden, CT, USA) for determination of C-5 MT-ase activity. Each reaction was performed in triplicate and the assay was repeated three times to blind the source of the samples. In order to exclude background protein, all samples were initially assayed with the control containing the whole cell lysate, but without poly(deoxyinosinic-deoxycytidylic) acid. C-5 MT-ase activity was expressed as the quantity of incorporated $\left[\right.$ methyl $\left.-{ }^{3} \mathrm{H}\right]$ groups into the poly(deoxyinosinic-deoxycytidylic) acid (cpm/1 $\mu \mathrm{g}$ protein).

Statistical analysis. Each experiment was repeated three times. Results are expressed as the mean \pm standard deviation. Statistical comparisons of single parameters between two groups were performed using the paired Student's t-test. Kruskal-Wallis one-way analysis of variance was used to compare the means of multiple groups, followed by the Dunn test. A value of $\mathrm{P} \leq 0.05$ was considered to indicate a statistically significant difference.

\section{Results}

Hcy increases VSMC viability and stimulates the cell cycle. The primary culture of confluent VSMCs exhibited a typical elongated ribbon or spindle-shaped appearance with a characteristic 'hill and valley' pattern (Fig. 1A), and immunocytochemistry revealed $98 \%$ positive $\alpha$-actin staining (Fig. 1B). MTT assay showed that VSMC viability significantly increased by 1.2-, 2.2-, 3.6- and 4.3-fold subsequent to treatment with 50,100, 200 and $500 \mu \mathrm{M}$ Hcy, respectively, compared with the control group $(\mathrm{P}<0.01)$. Furthermore, folate treatment had an antagonistic effect against Hcy-induced VSMC proliferation, with a $63 \%$ decrease in VSMC viability observed in the folate plus $500 \mu \mathrm{M}$ Hcy group compared with the $500 \mu \mathrm{M}$ Hcy group $(\mathrm{P}<0.01)$ (Fig. 1C).

Flow cytometry revealed that, as Hcy concentration increased, the proportion of the cell population in $\mathrm{S}$ phase increased, and that in $\mathrm{G}_{0} / \mathrm{G}_{1}$ phase decreased. Folate showed an inhibitory effect against Hcy on the VSMC cell cycle (Fig. 2).

Hcy increases PDGF $m R N A$ and protein expression. qPCR and western blot analyses revealed a dose-dependent increase in PDGF mRNA and protein expression with Hcy treatment, in parallel with the increase in VSMC proliferation observed under Hcy treatment. PDGF has a key role in VSMC prolif- 

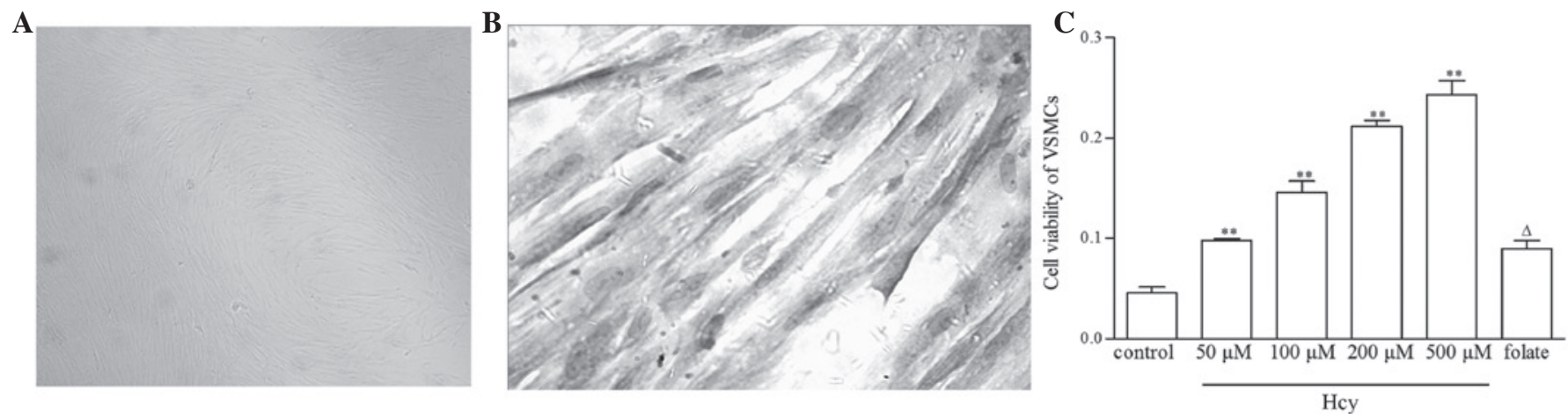

Figure 1. VSMC primary culture and viability assay. (A) Primary VSMCs cultured for 15 days (magnification, x200). (B) Cultured primary VSMCs verified using $\alpha$-actin immunocytochemistry (magnification, x400). (C) Effect of Hcy on cell viability assessed using MTT assay. Data are expressed as the mean \pm standard deviation. ${ }^{* *} \mathrm{P}<0.01$ vs. control group; ${ }^{\circ} \mathrm{P}<0.01$ vs. $500 \mu \mathrm{M}$ Hcy group. VSMC, vascular smooth muscle cell; Hcy, homocysteine.

A
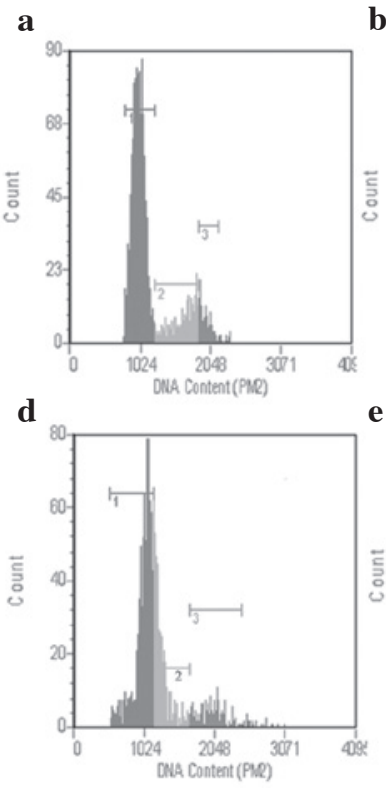

b
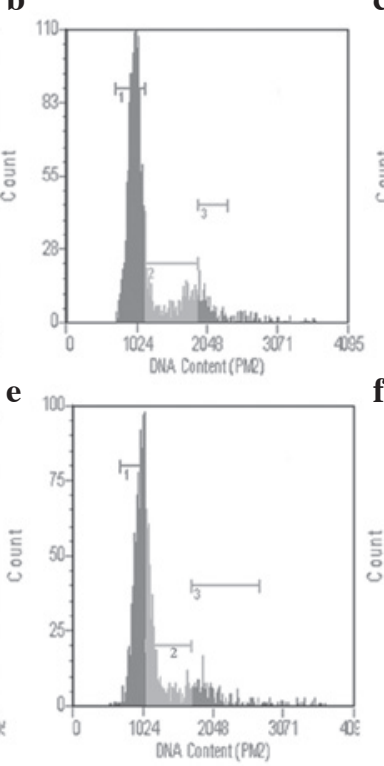

c
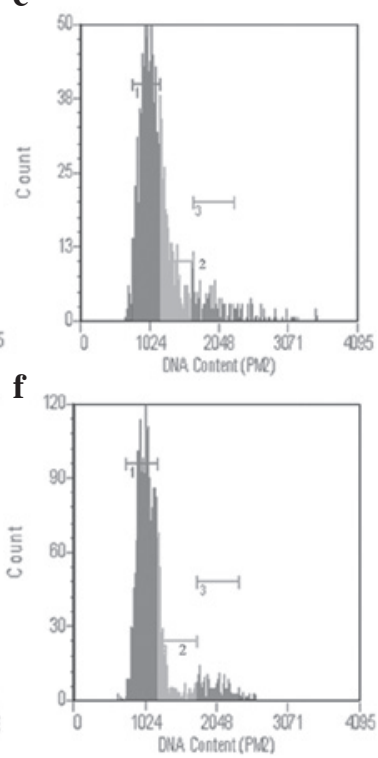

B

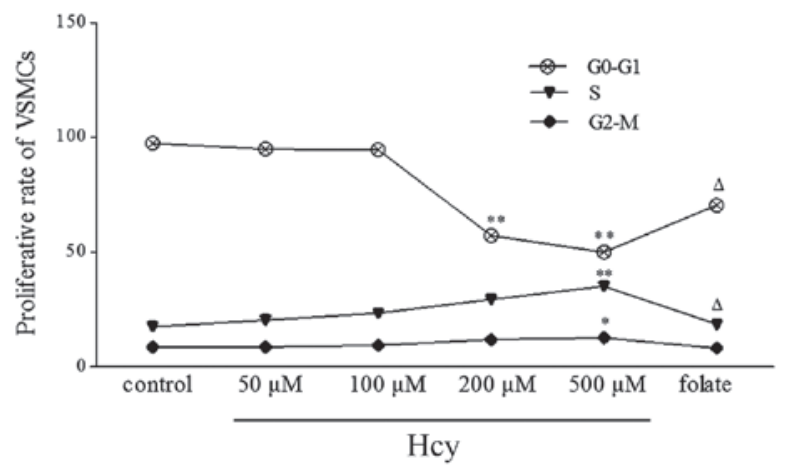

Figure 2. VSMC cell cycle phases following Hcy treatment, detected using flow cytometry. (A) Representative flow cytometric profiles demonstrating VSMC cell cycle distribution. (a) 0 , (b) 50, (c) 100 , (d) 200 and (e) $500 \mu \mathrm{M} \mathrm{Hcy} ; 1, \mathrm{G}_{0} / \mathrm{G}_{1}$ phase; $2, \mathrm{~S}$ phase; $3, \mathrm{G}_{2} / \mathrm{M}$ phase. (B) Summary of cell cycle analysis, revealing an increased $S$ fraction and a decreased $G_{0} / G_{1}$ fraction in Hcy-treated VSMCs. The values for each experiment were converted to percentages relative to the corresponding control group $(100 \%)$. Values are presented as the mean \pm standard deviation. ${ }^{*} \mathrm{P}<0.05$ and ${ }^{* * *} \mathrm{P}<0.01$ vs. control group; ${ }^{\wedge} \mathrm{P}<0.05$ vs. $500 \mu \mathrm{M}$ Hcy group. VSMC, vascular smooth muscle cell; Hcy, homocysteine.

eration; therefore, this parallel alteration in VSMC PDGF expression and proliferation suggests a causative role for PDGF in Hcy-induced VSMC proliferation (Fig. 3).

Hcy induces hypomethylation of the PDGF gene. DNA methylation occurs almost exclusively at $\mathrm{CpG}$ dinucleotides, and
CpG methylation is often associated with gene silencing and vice versa. The DNA methylation level of the $\mathrm{CpG}$ islands in the PDGF gene promoter was analyzed using nMS-PCR. The PDGF gene promoter was found to be significantly hypomethylated in a dose-dependent manner upon Hcy treatment (Fig. 4). These data indicate that Hcy treatment inhibits 


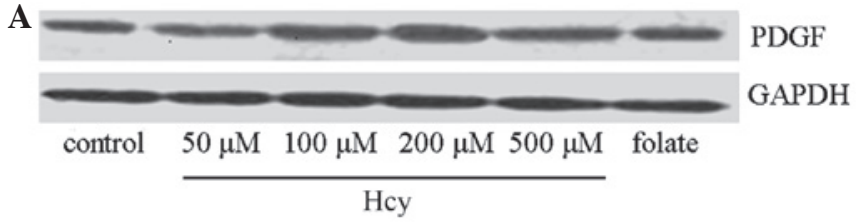

B

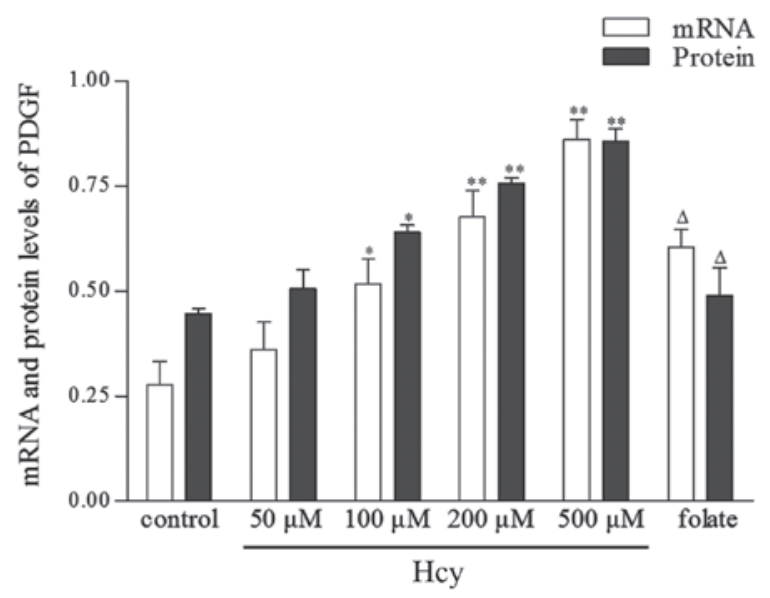

Figure 3. PDGF mRNA and protein expression in vascular smooth muscle cells detected using quantitative polymerase chain reaction and western blot analyses, respectively. Upon treatment with Hcy, a dose-dependent increase was observed in the (A and B) protein and (B) mRNA levels of PDGF. Values are presented as the mean \pm standard deviation. ${ }^{*} \mathrm{P}<0.05$ and ${ }^{* *} \mathrm{P}<0.01$ vs. control group; ${ }^{\triangle} \mathrm{P}<0.01$ vs. $500 \mu \mathrm{M}$ Hcy group. Hcy, homocysteine; PDGF, platelet-derived growth factor.

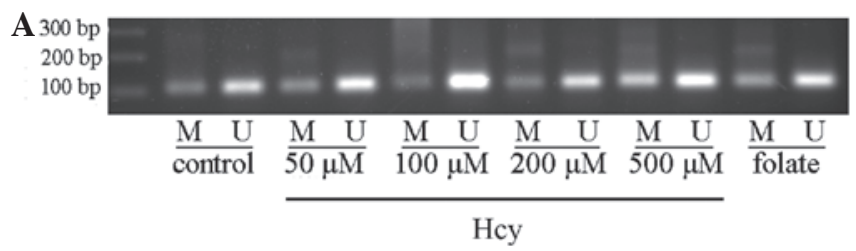

B

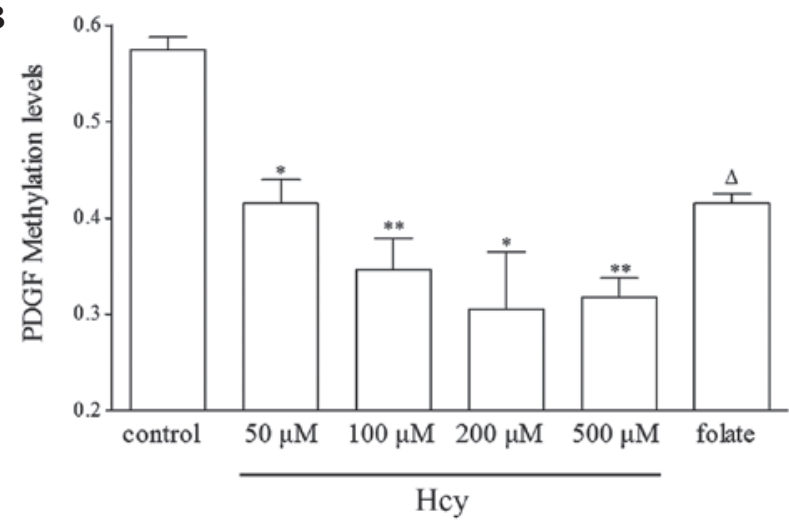

Figure 4. PDGF methylation levels subsequent to treatment with Hcy in VSMCs. (A) PDGF methylation detected using nested methylation-specific-polymerase chain reaction. (B) Summary of PDGF methylation levels in Hcy-treated VSMCs. Values are presented as the mean \pm standard deviation; ${ }^{*} \mathrm{P}<0.05$ and ${ }^{* *} \mathrm{P}<0.01$ vs. control group; ${ }^{\circ} \mathrm{P}<0.01$ vs. $500 \mu \mathrm{M}$ Hcy group. VSMC, vascular smooth muscle cell; Hcy, homocysteine; PDGF, platelet-derived growth factor; $\mathrm{M}$, methylated DNA; U, unmethylated DNA.

the methylation of the PDGF gene, and this demethylation effect may be involved in the upregulation of PDGF expression and VSMC proliferation in the pathogenesis of AS.
Hcy affects SAM and SAH levels and C-5 MT-ase activity. SAM and SAH concentrations are important factors in the transmethylation process. In the present study, SAM and SAH levels were assessed using HPLC. In the Hcy-treated groups, the intracellular levels of SAH were significantly higher than those in the control group, showing up to a three-fold increase. However, upon Hcy treatment the concentration of SAM was observed to decrease, leading to a significant decrease in the ratio of SAM/SAH. SAM is a primary methyl-donor while SAH is a potent inhibitor of methyltransferase activity; therefore, the SAM/SAH ratio may be critical in the modification of DNA methylation. However, the changes in SAM and SAH levels did not exhibit the dose-effect trend with Hcy concentration (Fig. 5).

C-5 MT-ase activity is another key regulator of the transmethylation reaction. Hcy was found to significantly upregulate the activity of C-5 MT-ase compared with the control cells (Fig. 6); however, no significant dose-dependent increase was observed.

\section{Discussion}

This study has indicated that a significant association exists among Hcy concentration, PDGF promoter hypomethylation, upregulated PDGF expression and cell proliferation in VSMCs. Despite the lack of direct evidence, the data of the present study suggest a causative interrelation among these processes. To the best of our knowledge, this is the first study to suggest that Hcy-induced VSMC proliferation may be mediated by epigenetic regulation of PDGF by Hcy.

VSMC proliferation is considered to be one of the important pathological factors in AS (19). AS begins with eccentric thickening of the intima, leading to complex lesions over several decades (20). The thickening neointima is predominantly composed of VSMCs, mesenchymal intimal cells and inflammatory cells (21). The pathogenesis of AS primarily involves changes in the expression and function of genes, rather than gene mutations, and the proliferation of VSMCs requires significant alterations in gene expression, particularly in mitogenic genes (22). Numerous studies, including our previous study (23), have demonstrated that Hcy is capable of stimulating VSMC proliferation (24). In the present study, PDGF mRNA and protein levels showed a dose-dependent increase with Hcy treatment, and cell cycle analysis further revealed an increased proportion of cells advancing into S phase from $G_{0} / G_{1}$-phase, suggesting that VSMCs proliferated under Hcy treatment.

It is well established that PDGF is a potent mitogenic factor, and has a critical role in normal embryonic development, cellular differentiation and proliferation (25). Increased PDGF expression subsequent to arterial injury has been associated with neointimal cellular proliferation in studies of rabbits, birds, rats and humans (26-29), demonstrating an association between VSMC proliferation and PDGF. Furthermore, the pathological processes that occur in proliferative diseases, such as AS, have been successfully suppressed by inhibitors of PDGF signaling (30). Sirois et al (31) demonstrated that inhibition of PDGF suppressed intimal thickening in the rat carotid artery following balloon injury. Additionally, the administration of anti-PDGF antibodies has been found to induce intimal atrophy in a baboon graft model (32). These observations 
A

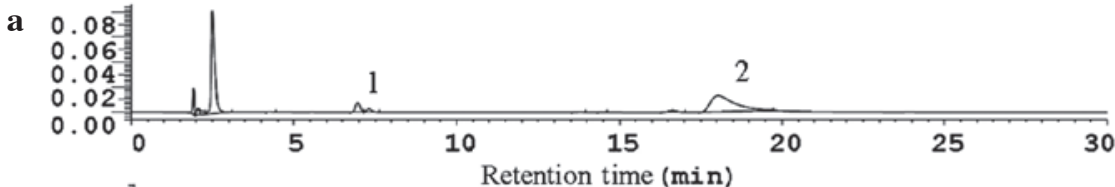

b
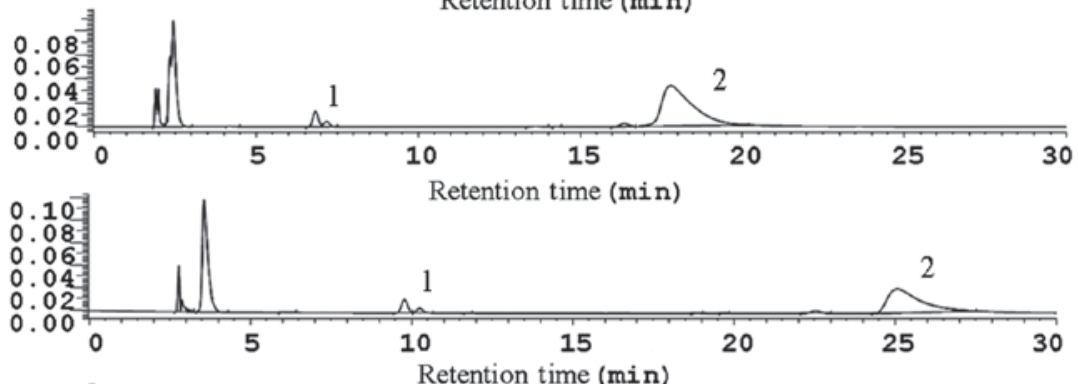

d 0.04

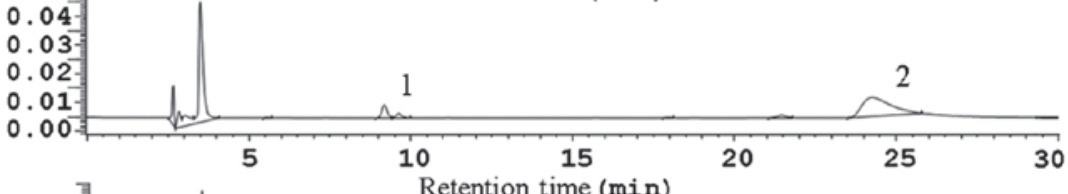

e 0.04

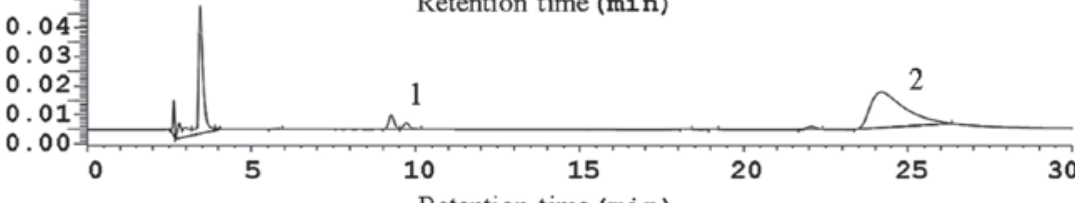

B

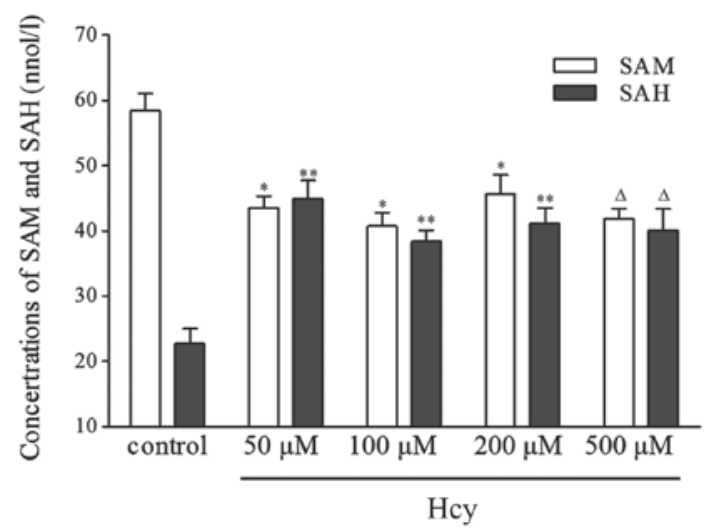

C

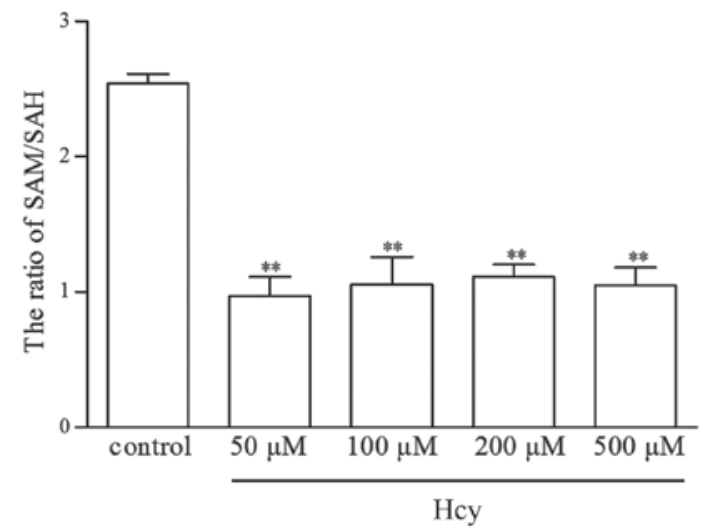

Figure 5. Effect of Hcy treatment on SAM and SAH concentrations in VSMCs (A) Chromatogram of SAH and SAM detected using high-performance liquid chromatography. (a) 0, (b) 50, (c) 100, (d) 200 and (e) $500 \mu$ M Hcy. Peaks: 1, SAM; 2, SAH. (B) Effects of Hcy on SAH and SAM in VSMCs. SAM and SAH quantification was performed using automatic peak area integration. SAM and SAH standards were used to identify the elution peaks. (C) Ratio of SAM and SAH. Values are presented as the mean \pm standard deviation. ${ }^{*} \mathrm{P}<0.05$ and ${ }^{* *} \mathrm{P}<0.01$ vs. control group; ${ }^{\triangle} \mathrm{P}<0.01$ vs. $500 \mu \mathrm{M}$ Hcy group. SAM, S-adenosylmethionine; SAH, S-adenosylhomocysteine; VSMC, vascular smooth muscle cell; Hcy, homocysteine.

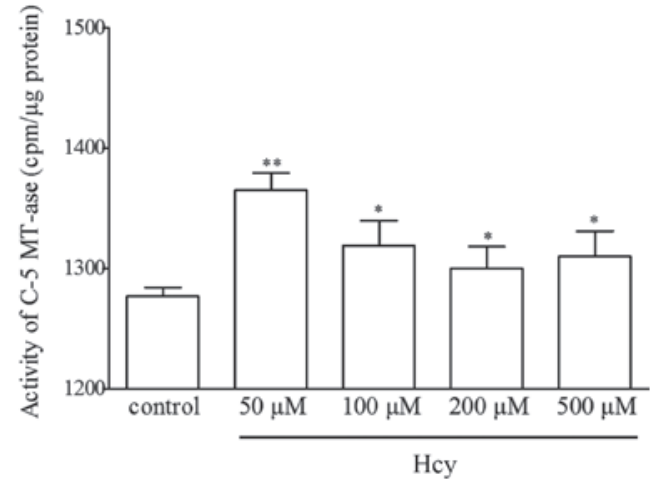

Figure 6. Endogenous C-5 MT-ase activity in vascular smooth muscle cells treated with various homocysteine concentrations. Data are presented as the mean \pm standard deviation of three independent experiments with three simultaneous samples each. ${ }^{*} \mathrm{P}<0.05$ and ${ }^{* *} \mathrm{P}<0.01$ vs. control group. C-5 MT-ase, C-5 DNA methyltransferase. support a pathogenic role for PDGF in proliferative vascular diseases (33). In the present study, Hcy was observed to induce a parallel alteration in PDGF expression and VSMC proliferation, suggesting that Hcy may stimulate VSMC proliferation through the PDGF signaling pathway.

In the present study, PDGF promoter methylation was detected in order to investigate the mechanism underlying Hcy-induced PDGF overexpression. A positive, dose-dependent correlation was identified between Hcy concentration, decreased methylation of the PDGF promoter and upregulation of PDGF expression. DNA methylation is a key form of the epigenetic genomic modification that regulates gene expression, and hypomethylation of the $\mathrm{CpG}$ islands in gene promoter regions is often associated with increased gene expression. Therefore, Hcy-induced PDGF hypomethylation may be responsible for the increased PDGF expression. 
DNA methylation status is significantly correlated with the ratio of SAM/SAH (34). In the present study, a decrease in the SAM/SAH ratio was observed upon Hcy treatment, which may partially explain the demethylation effect of Hcy on the PDGF gene. In the methionine cycle, which connects Hcy metabolism and the transmethylation reaction of DNA, SAH is hydrolyzed to form Hcy; therefore, increased levels of Hcy inhibit the decomposition of SAH, resulting in $\mathrm{SAH}$ accumulation. SAH is a potent inhibitor of SAM-dependent methyltransferases (9); therefore, increased levels of SAH may promote genomic demethylation, while decreased levels of SAM, which is the sole methyl-donor for DNA methylation, may limit its availability and coordinate the demethylation effect of SAH. Hiltunen et al (35) showed that only a few rounds of replication are required to develop significant hypomethylation of the smooth muscle cell genome; the decrease in the SAM/SAH ratio may thus be an important mechanism for PDGF hypomethylation in VSMCs. The increase in C-5 MT-ase activity observed in the study may be a compensatory response against the inhibition of SAH in this in vitro experiment.

In the present study, folate supply exhibited an antagonistic effect against the Hcy-induced aberrant PDGF methylation, increase in PDGF expression and increase in VSMC proliferation. In the methionine cycle, Hcy is converted to methionine by acquiring a methyl group from N-5-methyltetrahydrofolate, a reaction catalyzed by methionine synthase (36). Methionine is then activated to SAM. Folate increases the production of $\mathrm{N}-5$-methyltetrahydrofolate, promotes the transformation of Hcy to SAM and decreases levels of Hcy and SAH. Therefore, the antagonistic effect of folate against Hcy is closely associated with the methionine cycle. This is consistent with the effect of folate on the methylation status of the PDGF gene and VSMC proliferation observed in the present study. Hcy was found to interfere with the epigenetic regulation of PDGF gene expression; since the epigenetic regulation of PDGF gene expression may be involved in VSMC proliferation, these findings may be beneficial for patients with cardiovascular disorders and AS.

In conclusion, the present study has demonstrated that Hcy-induced VSMC proliferation may be mediated through PDGF signaling by interference with the epigenetic regulation of PDGF. Hcy induces hypomethylation of the promoter region of the PDGF gene and upregulates PGDF mRNA and protein expression, which eventually causes VSMC proliferation. These data may provide evidence for a useful target for the prevention and treatment of AS caused by Hcy.

\section{Acknowledgements}

This study was supported by grants from the National Natural Science Foundation of China (nos. 81260105 and 81200118), the Ningxia Education Department Scientific and Technological Project (nos. NGY2012056 and NGY2013082) and a grant from the Ningxia Science and Technique Project (no. NZ1195).

\section{References}

1. Zhang J, Guo C, Wang R, Huang L, Liang W, Liu R and Sun B An Egr-1-specific DNAzyme regulates Egr-1 and proliferating cell nuclear antigen expression in rat vascular smooth muscle cells. Exp Ther Med 5: 1371-1374, 2013.
2. Little PJ, Rostam MA, Piva TJ, et al: Suramin inhibits PDGF-stimulated receptor phosphorylation, proteoglycan synthesis and glycosaminoglycan hyperelongation in human vascular smooth muscle cells. J Pharm Pharmacol 65: 1055-1063, 2013.

3. Jia G, Cheng G, Gangahar DM and Agrawal DK: Involvement of connexin 43 in angiotensin II-induced migration and proliferation of saphenous vein smooth muscle cells via the MAPK-AP-1 signaling pathway. J Mol Cell Cardiol 44: 882-890, 2008.

4. Adhikari N, Basi DL, Townsend D, Rusch M, Mariash A, Mullegama S, Watson A, Larson J, Tan S, Lerman B, Esko JD, Selleck SB and Hall JL: Heparan sulfate Ndst1 regulates vascular smooth muscle cell proliferation, vessel size and vascular remodeling. J Mol Cell Cardiol 49: 287-293, 2010.

5. Luo X, Xiao Y, Song F, Yang Y, Xia M and Ling W: Increased plasma $S$-adenosyl-homocysteine levels induce the proliferation and migration of VSMCs through an oxidative stress-ERK1/2 pathway in apoE(-/-) mice. Cardiovasc Res 95: 241-250, 2012.

6. Zhang D, Chen Y, Xie X, Liu J, Wang Q, Kong W and Zhu Y: Homocysteine activates vascular smooth muscle cells by DNA demethylation of platelet-derived growth factor in endothelial cells. J Mol Cell Cardiol 53: 487-496, 2012.

7. Janda K, Aksamit D, Drozdz M, et al: Influence of elevated homocystein level and selected lipid parameters in kidney transplant patients on the progression of atherosclerotic changes assessed by intima-media thickness index (CCA-IMT). Przegl Lek 69: 670-674, 2012 (In Polish).

8. Huidobro C, Fernandez AF and Fraga MF: The role of genetics in the establishment and maintenance of the epigenome. Cell Mol Life Sci 70: 1543-1573, 2013.

9. Tisdale MJ: Potentiation of the growth inhibitory effects of adenosine 3',5'-monophosphate analogues by homocysteine. Biochem Pharmacol 31: 979-982, 1982.

10. Zhang CY, Wang NN, Zhang YH, Feng QZ, Yang CW and Liu B: DNA methylation involved in proline accumulation in response to osmotic stress in rice (Oryza sativa). Genet Mol Res 12: 1269-1277, 2013

11. He Y, Cui Y, Wang W, Gu J, Guo S, Ma K and Luo X: Hypomethylation of the hsa-miR-191 locus causes high expression of hsa-mir-191 and promotes the epithelial-to-mesenchymal transition in hepatocellular carcinoma. Neoplasia 13: 841-853, 2011

12. Amatruda JF, Ross JA, Christensen B, et al: DNA methylation analysis reveals distinct methylation signatures in pediatric germ cell tumors. BMC Cancer 13: 313, 2013

13. Yideng J, Zhihong L, Jiantuan X, Jun C, Guizhong L and Shuren W: Homocysteine-mediated PPARalpha,gamma DNA methylation and its potential pathogenic mechanism in monocytes. DNA Cell Biol 27: 143-150, 2008.

14. Sahni A, Wang N and Alexis J: UAP56 is an important mediator of angiotensin II/platelet derived growth factor induced vascular smooth muscle cell DNA synthesis and proliferation. Biochem Biophys Res Commun 431: 636-640, 2013.

15. Thevathasan JV, Tan E, Zheng H, Lin YC, Li Y, Inoue T and Fivaz M: The small GTPase HRas shapes local PI3K signals through positive feedback and regulates persistent membrane extension in migrating fibroblasts. Mol Biol Cell 24: 2228-2237, 2013.

16. Chandra A and Angle N: VEGF inhibits PDGF-stimulated calcium signaling independent of phospholipase $\mathrm{C}$ and protein kinase C. J Surg Res 131: 302-309, 2006.

17. Kojima N, Hori M, Murata T, Morizane Y and Ozaki H: Different profiles of $\mathrm{Ca}^{2+}$ responses to endothelin-1 and PDGF in liver myofibroblasts during the process of cell differentiation. $\mathrm{Br} \mathrm{J}$ Pharmacol 151: 816-827, 2007.

18. Hattori N, Abe T, Hattori N, et al: Preference of DNA methyltransferases for $\mathrm{CpG}$ islands in mouse embryonic stem cells. Genome Res 14: 1733-1740, 2004.

19. Dai Y, Mercanti F, Dai D, Wang X, Ding Z, Pothineni NV and Mehta JL: LOX-1, a bridge between GLP-1R and mitochondrial ROS generation in human vascular smooth muscle cells. Biochem Biophys Res Commun 437: 62-66, 2013.

20. Whitman SC: A practical approach to using mice in atherosclerosis research. Clin Biochem Rev 25: 81-93, 2004.

21. Shi ZD and Tarbell JM: Fluid flow mechanotransduction in vascular smooth muscle cells and fibroblasts. Ann Biomed Eng 39: 1608-1619, 2011. 
22. Xie C, Ritchie RP, Huang H, Zhang J and Chen YE: Smooth muscle cell differentiation in vitro: models and underlying molecular mechanisms. Arterioscler Thromb Vasc Biol 31: 1485-1494, 2011

23. Yideng J, Jianzhong Z, Ying H, Juan S, Jinge Z, Shenglan W, Xiaoqun $\mathrm{H}$ and Shuren W: Homocysteine-mediated expression of SAHH, DNMTs, MBD2, and DNA hypomethylation potential pathogenic mechanism in VSMCs. DNA Cell Biol 26: 603-611, 2007.

24. Liu X, Shen J, Zhan R, et al: Proteomic analysis of homocysteine induced proliferation of cultured neonatal rat vascular smooth muscle cells. Biochim Biophys Acta 1794: 177-184, 2009.

25. Nazarenko I, Hede SM, He X, Hedrén A, Thompson J, Lindström MS and Nistér M: PDGF and PDGF receptors in glioma. Ups J Med Sci 117: 99-112, 2012.

26. Sakata Y, Xiang F, Chen Z, Kiriyama Y, Kamei CN, Simon DI and Chin MT: Transcription factor $\mathrm{CHF} 1 / \mathrm{Hey} 2$ regulates neointimal formation in vivo and vascular smooth muscle proliferation and migration in vitro. Arterioscler Thromb Vasc Biol 24: 2069-2074, 2004.

27. Zhang H, Jia X, Han F, Zhao J, Zhao Y, Fan Y and Yuan X: Dual-delivery of VEGF and PDGF by double-layered electrospun membranes for blood vessel regeneration. Biomaterials 34: 2202-2212, 2013.

28. Li JC, Pan JQ, Huang GQ, Tan X, Sun WD, Liu YJ and Wang XL: Expression of PDGF-beta receptor in broilers with pulmonary hypertension induced by cold temperature and its association with pulmonary vascular remodeling. Res Vet Sci 88: 116-121, 2010 .
29. Takimoto T, SuzukiK,Arisaka H,Murata T,OzakiH and KoyamaN: Effect of N-(p-coumaroyl)serotonin and $\mathrm{N}$-feruloylserotonin, major anti-atherogenic polyphenols in safflower seed, on vasodilation, proliferation and migration of vascular smooth muscle cells. Mol Nutr Food Res 55: 1561-1571, 2011.

30. Choi BK, Cha BY, Yagyu T, Woo JT and Ojika M: Sponge-derived acetylenic alcohols, petrosiols, inhibit proliferation and migration of platelet-derived growth factor (PDGF)-induced vascular smooth muscle cells. Bioorg Med Chem 21: 1804-1810, 2013.

31. Sirois MG, Simons M and Edelman ER: Antisense oligonucleotide inhibition of PDGFR-beta receptor subunit expression directs suppression of intimal thickening. Circulation 95: 669-676, 1997.

32. Englesbe MJ, Hawkins SM, Hsieh PC, Daum G, Kenagy RD and Clowes AW: Concomitant blockade of platelet-derived growth factor receptors alpha and beta induces intimal atrophy in baboon PTFE grafts. J Vasc Surg 39: 440-446, 2004.

33. Kwon HJ, Kim GE, Lee YT, Jeong MS, Kang I, Yang D and Yeo EJ: Inhibition of platelet-derived growth factor receptor tyrosine kinase and downstream signaling pathways by Compound C. Cell Signal 25: 883-897, 2013.

34. Chen NC, Yang F, Capecci LM, et al: Regulation of homocysteine metabolism and methylation in human and mouse tissues. FASEB J 24: 2804-2817, 2010.

35. Hiltunen MO, Turunen MP, Häkkinen TP, et al: DNA hypomethylation and methyltransferase expression in atherosclerotic lesions. Vasc Med 7: 5-11, 2002.

36. Duncan TM, Reed MC and Nijhout HF: The relationship between intracellular and plasma levels of folate and metabolites in the methionine cycle: a model. Mol Nutr Food Res 57: 628-636, 2013 